\title{
¿Por qué el propofol en el paciente con cáncer?
}

\section{¿Why propofol in the cancer patient?}

\author{
Guillermo Capote Guerrero'
}

\begin{abstract}
Introduction: Cancer is a chronic, incapacitating and high mortality disease. Objective: To carry out an update on the beneficial properties of the use of propofol as an anesthetic agent in cancer patients. Methodological design: A manual and digital search was performed in different databases such as MEDLINE, Scielo, IBECS and Cochrane, with the following descriptors: propofol, cancer, immunity and cancer. Development: $90 \%$ of deaths related to cancer are due to the progression of the disease, to metastases and not to the primary tumor. Therefore, preventing immunosuppression in the perioperative period is particularly important. During the perioperative period, there are factors that activate or inhibit immune responses. Recent findings have suggested that anesthesia can induce metabolic, inflammatory and immunological changes in the perioperative period. Propofol promotes the cytotoxicity of natural killer cells, reduces the motility of tumor cells, inhibits cyclo-oxygenase. It has been revealed that this drug exhibits anticancer properties in some types of cancer: colon cancer, gastric cancer, bile duct cancer. Conclusions: Propofol has a potential benefit as an anesthetic agent in patient with cancer. Only the accumulation of even more scientific evidence would allow us to give greater value to the use of this drug.
\end{abstract}

\section{RESUMEN}

Introducción: El cáncer es una enfermedad crónica, incapacitante y de gran mortalidad. Objetivo: Realizar una actualización sobre las beneficiosas propiedades del uso del propofol como agente anestésico en el paciente oncológico. Diseño metodológico: Se realizó una búsqueda manual y digital en diferentes

\section{Key words:}

Propofol, anesthesia, cancer, immunity

\section{Palabras clave:}

Propofol, anestesia, cáncer, inmunidad

Profesor auxiliar. Especialista de segundo grado en anestesiología y reanimación. Diplomado en terapia intensiva y emergencias. Hospital Carlos Manuel de Céspedes. Bayamo, Granma.

Fecha de ingreso: 21 de septiembre de 2018

Fecha de aceptación: 24 de noviembre de 2018

\section{ORCID}

https://orcid.org/0000-0003-2841-8348

Correspondencia:

Guillermo Capote Guerrero

E-mail: gcapote82@gmail.com 
bases de datos como MEDLINE, Scielo, IBECS y Cochrane, con los descriptores siguientes: propofol, cáncer, inmunidad y cáncer. Desarrollo: El 90\% de las muertes relacionadas con el cáncer son debido a la progresión de la enfermedad, a las metástasis y no al tumor primario. Por ello, prevenir la inmunosupresión en el período perioperatorio toma particular importancia. Durante el periodo perioperatorio existen factores que activan o inhiben las respuestas inmunitarias. Los descubrimientos recientes han sugerido que la anestesia puede inducir cambios metabólicos, inflamatorios e inmunológicos en el período perioperatorio. El propofol favorece la citotoxicidad de las células naturalkiller, reduce la motilidad de las células tumorales, inhibe la ciclooxigenasa. Ha sido revelado que este fármaco exhibe propiedades anticancerosas en algunos tipos de cáncer: cáncer de colon, cáncer gástrico, cáncer de vías biliares. Conclusiones: El propofol presenta un potencial beneficio como agente anestésico en el paciente con cáncer. Solo bastaría la acumulación de aún más evidencia científica que nos permita darle mayor valor al uso de este fármaco.

\section{Introducción}

cáncer es una enfermedad crónica, incapacitante y de gran mortalidad[1].

El cáncer está reconocido como un importante problema de salud pública debido a la alta frecuencia de casos, a su impacto económico y a su trascendencia social[2]. Es actualmente la principal causa de muerte en todo el mundo. La Organización Mundial de la Salud (OMS) así como la Organización Panamericana de Salud (OPS) en su revisión de 2017, refiere que en el 2015 el cáncer ocasionó 8,8 millones de muertes a nivel mundial. Se destacan como los cánceres con mayor número de fallecimientos los siguientes: Pulmonar, Hepático, Colorrectal, Gástrico, Mamario[3].

Pero en América Latina la situación no es diferente. Según estadísticas de la OPS el cáncer es la segunda causa de muerte en la región. Se estima que 2,8 millones de personas son diagnosticadas cada año y 1,3 millones de personas mueren por esta enfermedad anualmente. Aproximadamente el $52 \%$ de los nuevos casos ocurren en personas de 65 años o menos[4],[5].

En Cuba, el cáncer es un grave problema de salud. Es considerado la segunda causa de muerte. Esta enfermedad en nuestro país, aunque mantiene el aumento de nuevos casos, la mortalidad comienza a tener una tendencia en meseta[5].

Extrapolando el incremento estadístico del cáncer en la especialidad de Anestesiología y Reanimación se concluye que el paciente oncológico es muy frecuente en los quirófanos. Se estima que un $30 \%$ de pacientes diagnosticados requieren algún tratamiento quirúrgico[6].
Un número creciente de publicaciones médicas avalan la hipótesis de que la respuesta al estrés quirúrgico y otros factores pudieran aumentar la probabilidad de diseminación y la metástasis durante el periodo perioperatorio en la cirugía del cáncer. El manejo anestésico del paciente oncológico, por lo tanto, podría influir en el resultado a largo plazo. Diversos estudios preclínicos sugieren que un enfoque beneficioso debería incluir una selección de diversos fárma$\cos [7]$.

Actualmente, un creciente número de estudios han demostrado que los anestésicos y la técnica anestésica tienen efectos beneficiosos en la recurrencia posoperatoria y puede incrementar la tasa de supervivencia en varios tipos de cáncer[7].

La literatura actual sugiere que la elección del anestésico está correlacionada con la supervivencia o la recurrencia del paciente oncológico después de la cirugía[8].

Constituye el objetivo de este artículo realizar una actualización sobre las beneficiosas propiedades del uso del propofol como agente anestésico en el paciente oncológico.

\section{Diseño metodológico}

Se realizó una búsqueda manual y digital en diferentes bases de datos como MEDLINE,Scielo, IBECS y Cochrane, con los descriptores siguientes: propofol, cáncer, inmunidad y cáncer. Al efecto, se encontraron diferentes artículos que versaban sobre el tema. De ellos se escogieron 29 publicados todos en los últimos 5 años. 


\section{Desarrollo}

El 90\% de las muertes relacionadas con el cáncer son debido a la progresión de la enfermedad, a las metástasis y no al tumor primario. Por ello, prevenir la inmunosupresión en el período perioperatorio toma particular importancia, ya que esto permitirá detener o por lo menos retrasar el crecimiento tumoral en este período de alto riesgo oncológico[2]. Además de que por definición el paciente con cáncer es un paciente inmunodeprimido[9].

Durante el período perioperatorio existen factores que activan o inhiben las respuestas inmunitarias.

- Respuesta neuroendocrina: liberación de catecolaminas (CAT) que se unen a los receptores $\beta$ de las células tumorales, estimulando el crecimiento y la diseminación tumoral y la angiogénesis. También se unen a los receptores $\beta 2$ de las células citotóxicas, disminuyendo la inmunidad celular (IC) [10].

- Activación del eje hipotálamo-hipofisarioadrenal: se produce una inmunosupresión por la liberación de glucocorticoides y CAT que inhiben la IC[10].

- Respuesta inflamatoria: se crea un microentorno tumoral con la liberación de citoquinas (CK), interleukinas (IL) y prostaglandinas (PG) que promueve la diseminación tumoral y la angiogénesis. La IL-2, la IL-12, el interferón- $\gamma$ (IFN- $\gamma$ ) y el factor de necrosis tumoral (TNF) estimulan la actividad de las células natural-killer (CNK) de la inmunidad celular. El resto de IL, sobre todo, la IL-6, la IL-8 y la IL-10 son inmunosupresoras al deprimir la IC. EI balance neto en el perioperatorio se inclina hacia la inmunosupresión[10].

- Inmunidad celular: las CNK son la primera línea de defensa antitumoral. Bajos niveles de actividad de las CNK en el perioperatorio son predictivos de alto riesgo de recidiva tumoral, independientemente de factores como la edad, el sexo, el tamaño, la diferenciación y el grado de invasión del tumor.

Las células T-helper pueden transformarse en Th1 o en Th2. Cuando el equilibrio se desvía hacia la producción de células Th2, se produce una disminución de la IC. Las células dendríticas y las células mononucleares, forman parte también de la IC. Se han descrito nuevas estirpes de linfocitos T citotóxicos (LT). Los LT marginales hepáticos y pulmonares, y los LT pit hepáticos. Estas células se localizan en las sinusoides de órganos como el hígado o el pulmón, presentando una gran capacidad de depuración de las células tumorales. A raíz de los nuevos descubrimientos de poblacio- nes linfocitarias citotóxicas, se cree que la IC juega un papel protagonista en la defensa antitumoral y anti-metastásica[10].

- Angiogénesis: el desarrollo de nuevas redes capilares es vital para el crecimiento tumoral. Los tumores no pueden sobrevivir más allá de los 2 $\mathrm{mm}$ de diámetro sin angiogénesis. Este proceso es estimulado por la liberación de factores proangiogénicos desde el tumor, incluyendo el factor vascular de crecimiento endotelial (VEGF) y la prostaglandina $E 2$, que también son activados por muchos factores pro-tumorales, como lo opioides. La acción anti-angiogénica se ejerce a través de la liberación de angiostatina y endostatina, que disminuyen tras la extirpación quirúrgica del tumor primario[10].

- Liberación de factores de crecimiento: durante la cirugía se produce una liberación de factores de crecimiento, migración y adherencia tumoral. Todos los anestésicos excepto el propofol activan el factor inducido por la hipoxia (HIF) que se localiza en el centro de la masa tumoral, y que promueve la proliferación y migración celular, la diseminación hematógena y la angiogénesis. Se plantea que el potencial efecto organoprotector de los anestésicos volátiles frente a la lesión de isquemia reperfusión, pueda jugar un papel negativo en la preservación de la inmunidad celular[10].

La cirugía para cánceres pone la mira en eliminar células cancerosas. Sin embargo, también puede promover la migración de las células del tumor, conduciendo a las metástasis. Los descubrimientos recientes han sugerido que la anestesia puede inducir cambios metabólicos, inflamatorios e inmunológicos en el período perioperatorio[11].

La función inmunitaria, sobre todo la inmunidad celular a expensas de las células NK (natural killer), juegan un papel fundamental en la recidiva tumoral y en la supervivencia. Hay un interés creciente en los eventos que se producendurante el período perioperatorio que conducen a la liberación de las células cancerosas en la circulación y que favorecen el crecimiento de las células tumorales circulantes, dando lugar a recurrencias regionales y metástasis. La técnica anestésica per se, también podría influir en los resultados oncológicos a largo plazo. Los fármacos anestésicos pueden ser capaces de inducir cambios biomoleculares involucrados en las funciones fisiopatológicas celulares, tales como la proliferación, la angiogénesis y la apoptosis celular, todos ellos, decisivos en la progresión de la enfermedad[12].

Las células natural Killer (NK) juegan un papel im- 
portante en la destrucción de células tumorales y restricción de crecimiento tumoral; éstas son disminuidas en presencia de estados pro-inflamatorios (incremento de interleucina 1 (IL-1), factor de necrosis tumoral alfa (TNF- $\alpha$ ) y PGE2. Esta última inhibe la función de las células dendríticas), al inhibir la actividad citotóxica de las NK y altera el balance entre la proliferación y erradicación de las células neoplásicas[13].

Los agentes anestésicos modulan varias áreas del sistema inmune, el sistema endocrino y la respuesta al estrés que inevitablemente acompaña a la cirugía[13].

La anestesia altera numerosas funciones inmunes, incluyendo las de neutrófilos, macrófagos, células dendríticas, células T y las células naturales killer (NK) [12].

Algunos de los fármacos utilizados frecuentemente en la anestesia general tienen un efecto inhibitorio de la inmunidad mediada por células natural-killer, particularmente la morfina, ketamina, tiopental y anestésicos volátiles[14].

Los datos in vitro del posible papel prometastásico de los anestésicos inhalatorios se basan en sus efectos inhibitorios tiempo y dosis-dependiente de la función de los neutrófilos, la supresión de citocinas liberadas por las células mononucleares, la disminución de la proliferación linfocitaria y el efecto favorecedor sobre la inducción de la apoptosis linfocitaria. Además de sus efectos sobre el sistema inmune los anestésicos inhalatorios también promueven el crecimiento y proliferación de las células madre del endotelio (efecto proangiogénico) que favorecería el crecimiento tumoral y/o lateratogenicidad[12].

Los agentes volátiles inhiben la estimulación con interferón de la citotoxicidad de las células NK en modelos animales. Reduce el número de células NK en los seres humanos y se asocia con peores resultados en comparación con anestesia local para la extirpación del melanoma[14].

El óxido nitroso se asocia con la aceleración en el desarrollo de cáncer de pulmón y metástasis en el hígado en modelos animales. Ningún efecto sobre el resultado del cáncer después de la cirugía para el carcinoma colorrectal en los seres humanos. Inhibe la formación de las células hematopoyéticas que pueden ser importantes para las células tumorales[14].

Los analgésicos opioides que juegan un importante papel como promotores deprogresión tumoral debido a su efecto en la angiogénesis. Su principal participación es en los receptores $\mu$ opioides (MOR) encontrados en células del endotelio vascular, los cuales al ser activados generan angiogénesis dependiente, del factor de crecimiento endotelial vascular (VEGF) y por lo tanto, un aumento en la tasa de creci- miento tumoral. También se ha visto que tienen efecto inmunomodulador, inhibiendo tanto inmunidad celular como humoral. Se ha hablado del efecto que tiene la morfina estimulando la migración de células tumorales con potencial proliferación en células endoteliales[13]. Sin embargo, los opioides pueden reducir la respuesta de estrés al dolor y ofrecer algún beneficio[12]. No obstante a esto en la actualidad no existe un consenso sobre el rol de los opioides en la patología tumoral.

El tiopental reduce la actividad y número de células natural-killer (modelos animales)[14].

La ketamina hasta no hace mucho tiempo se planteaba que reduce la actividad y número de células natural-killer (modelos animales)[14], pero recientemente se sugiere que la ketamina es aún más atractiva, en especial para el tratamiento del paciente oncológico, por la inhibición del factor nuclear-kB y el factor de la proteína 1, la inhibición de la activación de los neutrófilos, la inhibición de la producción de óxido nítrico producida por los macrófagos, la regulación proinflamatoria del óxido sintetasa y la ciclooxigenasa 2, además del bloqueo de los canales de larga conductancia Kca en la microglía. Lo anterior hace que el uso de ketamina en el paciente oncológico tenga resultados favorecedores en la inmunidad y, por lo tanto, en el proceso de metástasis y recurrencia de enfermedad[15]. Todo esto, a dosis subanestésicas. Estamos hablando de 0,1 a 0,3 mg/kg por hora.

Aunque todo esto se está planteando, en la actualidad consideramos que aún la evidencia es insuficiente como para hacer recomendaciones sobre que su uso sea beneficioso en pacientes oncológicos, y si deberíamos estar prestos a investigaciones futuras sobre el uso de este fármaco en el cáncer.

Al parecer, no todos los fármacos anestésicos deprimen el sistema inmunológico[16].

EI propofol es un agente hipnótico y sedativo endovenoso que se utiliza para la inducción y mantenimiento de la anestesia[17], ampliamente utilizado para hipnosis y sedación durante y después de las intervenciones quirúrgicas. Su efecto anestésico es debido a la activación directa de los receptores GABA y por bloqueo de los canales de sodio[18],[19].

El propofol favorece la citotoxicidadde las células natural killer (NK), reduce la motilidad de las células tumorales, inhibe la ciclooxigenasa y no promueve la síntesis de HIF (efecto antiangiogénico)[16].

Recientemente, ha sido revelado que este fármaco exhibe propiedades anticancerosas en algunos tipos de cáncer: cáncer de colon[20], cáncer gástrico[21], cáncer de vías biliares[22].

En un estudio publicado en el año 2018 en la Bra- 
zilian Journal of Medical and Biological Resecar[7], se demuestra que el propofol inhibe la proliferación, migración e invasión celular en el paciente con cáncer endometrial. Es capaz de inhibir la viabilidad he induce la apoptosis en el cáncer de pulmón de células no pequeñas[17].

En una investigación realizada en China[23] se comprobó que comparado con el sevofluorano la anestesia con propofol puede reducir significativamente la respuesta inflamatoria en pacientes a los cuales se le realizó resección pulmonar por cáncer. Así como disminución del índice de prevalencia de reacciones adversas intraoperatorias y queprotege la función pulmonar.

En un artículo publicado recientemente en la revista Cellular Physiology and Biochemistry[24] se demuestra que el propofol tiene un papel inhibitorio en la glucolisis de células de cáncer colorrectal. Se sugiere además que inhibie la expresión de transportadores de glucosa y enzimas (GLUT1, HK2, LDHA, y PDK1) que son cruciales en el metabolismo de la glucosa. Más allá mantuvo la hipótesis que HIF1, un factor maestro de la transcripción controlando metabolismo de glucosa, es también disminuido por el propofol en una manera dosis dependiente[24].

Un reciente análisis retrospectivo (Wigmore et al), de más de 7.000 pacientes de un hospital oncológico en el Reino Unido, fue llevado a cabo, para determinar la supervivencia a largo plazo en pacientes que recibieron anestesia inhalatoria (isofluorano o sevofluorano) versus anestesia intravenosa (propofol y remifentanilo) para operaciones de cáncer.

Después de un análisis de propensión y un análisis multivariable encontraron que aquellos que se les administró anestésicos inhalatorios tuvieron una menor supervivencia (HR [hazard ratio] de 1,46; IC 95\% 1,29$1,66)$ comparado con los que se utilizó anestesia intravenosa[25].

Zheng et al[26]. Publicaron en el 2018 un estudio retrospectivo de pacientes operados por cáncer gástrico entre el 2007 y el 2012, en este estudio se incluyeron 2.856 individuos repartidos en 2 grupos, uno en el cual se realizó el mantenimiento anestésico con propofol más remifentanil y el otro con sevoflurano y remifentanil, demostrándose una mayor supervivencia en los que se utilizó TIVA.

Un estudio in vivo evaluó el efecto del propofol en las metástasis de cáncer pulmonar. Después de la administración de propofol, 40 ratas fueron inoculadas con células de cáncer pulmonar, tres semanas des- pués se encontró que la dosis de propofol fue inversamente correlacionada con el número de metástasis pulmonares. De manera similar, el propofol inhibió drásticamente elcrecimiento de tumores hepatocelulares en modelos de ratas[27].

Hasta ahora, sólo existían estudios descriptivos entre diversas técnicas, que generalmente obtenían mejores resultados con anestesia combinada con anestesia regional y multimodal que atribuían al ahorro de opioides; pero no del efecto antitumoral del propofolen la clínica, ni tampoco sobre la supervivencia global y libre de metástasis o recurrencias en pacientes operados para practicarles mastectomía radical modificada por cáncer de mama. Parece que este fármaco no deprime el sistema inmunitario, según la evidencia disponible actualmente. El propofol favorece la citotoxicidad de las células NK, reduce la motilidad e invasividad de las células tumorales, inhibe la ciclooxigenasa y no promueve la síntesis de HIF (efecto antiangiogénico)[12].

Se ha demostrado que el propofol puede tener efectos protectores mediados por inhibición de COX2 y PGE-2 y favorece la actividad antitumoral, pero también mediante el incremento de la actividad antitumoral. El propofol es superior a los agentes volátiles en reducir el estrés quirúrgico que induce la supresión en la respuesta inmune. Estos resultados nos dan pie a pensar que la TIVA es preferible a los agentes inhalados en pacientes intervenidos quirúrgicamente por cáncer[14]. Además ejerce efectos antitumorales por la inhibición de la viabilidad de la célula, la dispersión y la aptitud de invasión de células de cáncer[28].

El potencial beneficio del propofol podría incluir el deterioro de la función de la célula cancerígena y la preservación de la función inmune[8].

Se reportó que el Propofol tiene actividad quimioterapéutica, evidenciado por su habilidad de inhibir la migración de las células tumorales[29].

El propofol es un anestésico intravenoso ampliamente usado en la anestesia general y en otras diversas situaciones médicas durante el tratamiento de los pacientes de cáncer[29]. Puede ser el mejor agente anestésico para la cirugía de cáncer comparado con otros anestésicos[17]

Se concluye que el propofol presenta un potencial beneficio como agente anestésico en el paciente con cáncer. Solo bastaría la acumulación de aún más evidencia científica que nos permita darle mayor valor al uso de este fármaco. 


\section{Referencias}

1. Pérez-Cruz E, Camacho-Limas CP. Cáncer del tracto digestivo: asociación entre el estado nutricional y la capacidad funcional. Gac Med Mex. 2017;153(5):575-80. https://doi. org/10.24875/GMM.17002776 PMID:29099098

2. Mille-Loera JE, Cuellar-Guzmán LP, Alvarado-Pérez J, GarcíaVelasco O, Fabela-Barragáncmas JA. Consideraciones anestésicas en el paciente con cáncer. Rev Mexicana de Anestesiologia.2017:40(1).132-4.

3. Cuba. Centro Nacional de Información de Ciencias Médicas. Biblioteca Médica Nacional. Tumores malignos. Tratamiento. Bibliomed [Internet]. 2017 May [Tumores malignos. Tratamiento. Bibliomed [Internet]. 2017 May [citado Día Mes Año];24(5):[aprox. 12 p.]. Disponible en: http://files.sld.cu/ bmn/files/2017/05/bibliomedmayo-2017.pdf

4. Organización Mundial de la Salud. Cáncer [Internet]. Fecha de consulta: Enero 20, 2018. Disponible en : http://www.paho.org/ hq/index.php?option=com_cont ent\&view=article\&id=292\%3Aca ncer-program \&catid $=1872 \% 3 \mathrm{Ac}$ ancer\&ltemid=3904\&lang=es

5. Arbesú AL. Un mundo sin cáncer para 2025: Nosotros podemos 2018. [Internet].Disponible en : http://www.cuba.cu/ salud/2018-02-03/un-mundosin-cancer-para-2025-nosotrospodemos/40005

6. Fuentes Díaz Z, Rodríguez Salazar O, Grau Ábalo R. Modelo pronóstico de mortalidad quirúrgica. Revista Cubana de Medicina Intensiva y Emergencias. 2018;17(1):60-70.

7. Du Q, Liu J, Zhang $X$, Zhang $X$, Zhu $\mathrm{H}$, Wei $\mathrm{M}$, et al. Propofol inhibits proliferation, migration, and invasion but promotes apoptosis by regulation of Sox 4 in endometrial cancer cells. Brazilian Journal of Medical and Biological Research. FapUNIFESP (SciELO); 2018 Feb 26;51(4). http://dx.doi.org/10.1590/1414431×20176803

8. Li R, Liu H, James P. Dilger, Jun Lin. Effect of Propofol on breast Cancer cell, the. immune system, and patient outcome [immune system, and patient outcome]. BMC Anesthesiol. 2018;18(77):18-77.

9. Reynoso NN, Mohar BA. Epidemiología del cáncer. En: Herrera GA, Namendys SSA, Meneses GA. Manual de Oncología procedimientos médico quirúrgico. Cap. 2, Sexta edición. McGrawHill. 2017; pp. 10-18.

10. Esteve N, Ferrer A, Mora C, Gómez G, Ribera H, Garrido P. ¿Influye la anestesia en los resultados de la cirugía oncológica? Revista de la Sociedad Española del Dolor. 2014;21(3):162. https://doi.org/10.4321/S113480462014000300007.

11. Ciechanowicz SJ, Ma D. Anaesthesia for oncological surgery - can it really influence cancer recurrence? Anaesthesia. 2016 Feb;71(2):127-31. https:// doi.org/10.1111/anae.13342 PMID:26669960

12. Bonilla Castillo L, Pérez Herrero MA, Torrent A. Efectos de la anestesia en la cirugía oncológica. Rev. Anestesiar. 2017;31(1):21-6.

13. Pierre C. Anestesia y Recurrencia: factores perioperatorios que favorecen recurrencia. Revista Mexicana de Anestesiología. 2015;38(1):314-7.

14. Cuéllar-Garduño N. Impacto de la técnica anestésica en la recurrencia del cáncer. Revista Mexicana de Anestesiología. 2014;37(1):296-8.

15. Vázquez Morales H, Cuéllar-Guz- mán LP, Arévalo-Villa D. La ketamina, una alternativa en el tratamiento anestésico del paciente oncológico. Gac Mex Oncol. 2018;17(2):114-20. https://doi. org/10.24875/j.gamo.18000077.

16. Bonilla-Castillo $L$, Pérez-Herrero MA, Abad-Torrent A. Efectos de la anestesia en la cirugía oncológica. GATIV/29 marzo 2017. Disponible en: https://anestesiar. org/2017/efectos-de-la-anestesia-inhalatoria-ointravenosasobre-la-supervivencia-o-recurrencia-en-cirugia-oncologica/

17. Xing SG, Zhang KJ, Qu JH, Ren YD, Luan Q. Propofol induces apoptosis of non-small cell lung cancer cells via ERK1/2dependent upregulation of PUMA. Eur Rev Med Pharmacol Sci. 2018 Jul;22(13):4341-9. PMID:30024623

18. García-Velasco O, Mille-Loera JE, Rocha-Machado JF. Propofol en el paciente con cáncer. Revista Mexicana de Anestesiología. 2018;41(1):125-7.

19. Sumi C, Okamoto A, Tanaka $H$, Nishi K, Kusunoki M, Shoji $\mathrm{T}$, et al. Propofol induces a metabolic switch to glycolysis and cell death in a mitochondrial electron transport chaindependent manner. PLoS One. 2018 Feb;13(2):e0192796. https://doi.org/10.1371/journal. pone.0192796 PMID:29447230

20. Xu YJ, Li SY, Cheng Q, Chen WK, Wang SL, Ren $Y$, et al. Effects of anaesthesia on proliferation, invasion and apoptosis of LoVo colon cancer cells in vitro. Anaesthesia. 2016 Feb;71(2):147-54. https:// doi.org/10.1111/anae.13331 PMID:26669824

21. Wang ZT, Gong HY, Zheng F, Liu DJ, Yue XQ. Propofol suppresses proliferation and invasion of gastric cancer cells via downregulation of microRNA-221 expression. Genet Mol Res. 2015 
Jul;14(3):8117-24. https://doi. org/10.4238/2015.July. 17.20 PMID:26214494

22. Zhang L, Wang N, Zhou S, Ye W, Jing G, Zhang M. Propofol induces proliferation and invasion of gallbladder cancer cells through activation of Nrf2. J Exp Clin Cancer Res. 2012 Aug;31(1):66. https://doi.org/10.1186/17569966-31-66 PMID:22901367

23. Tian $H T$, Duan $X H$, Yang $Y F$, Wang $Y$, Bai QL, Zhang X. Effects of propofol or sevoflurane anesthesia on the perioperative inflammatory response, pulmonary function and cognitive function in patients receiving lung cancer resection. Eur Rev Med Pharmacol Sci. 2017 Dec;21(23):5515-22. PMID:29243798

24. Chena X. Qichao Wua, Pengfei Suna, Yanjun Zhaoa, Minmin Zhua, Changhong
Miaoa. Propofol Disrupts Aerobic Glycolysis in Colorectal Cancer Cells via Inactivation of the NMDAR-CAMKII-ERK Pathway. Cell Physiol Biochem. 2018;46(2):492-504. https://doi. org/10.1159/000488617.

25. Wigmore TJ, Mohammed K, Jhanji S. Long-term Survival for Patients Undergoing Volatile versus IV Anesthesia for Cancer Surgery: A Retrospective Analysis. Anesthesiology. 2016 Jan;124(1):69-79. https://doi.org/10.1097/ ALN.0000000000000936 PMID:26556730

26. Zheng $X$, Wang $Y$, Dong $L$, Zhao S, Wang L, Chen $H$, et al. Effects of propofol-based total intravenous anesthesia on gastric cancer: a retrospective study. OncoTargets and Therapy. Dove Medical Press Ltd.; 2018 Mar;Volume 11:1141-8. http:// dx.doi.org/10.2147/ott.s156792

27. Yang W, Cai J, Zabkiewicz C, Zhang $H$, Ruge $F$, Jiang WG. The effects of anesthetics on recurrence and metastasis of cancer, and clinical implications. World J Oncol. 2017 Jun;8(3):63-70. https://doi.org/10.14740/wjon1031e PMID:29147437

28. Deng $F$, Ouyang $M$, Wang $X$, Yao X, Chen Y, Tao T, et al. Differential role of intravenous anesthetics in colorectal cancer progression: implications for clinical application. Oncotarget. 2016 Nov;7(47):77087-95. https://doi.org/10.18632/oncotarget.12800 PMID:27780923

29. Zhou Q, Tao T. Jun Fun, Cong Luo, Chen Zhu, Zai-Sheng Qin. Propofol inhibited invasion and decreased expression of PMEPA1 in MDA-MB-231 cells. Int J Clin Exp Med. 2017;10(10):14331-4. 